\title{
Principais causas de mortalidade na recria e terminação de bovinos de corte
}

\author{
Mortality rates and main causes in rearing and finishing cattle beef
}

\author{
Carlos Santos Gottschall ${ }^{[a]}$, Leonardo Canali Canellas ${ }^{[\mathrm{b}]}$, Marcos Rosa de Almeida ${ }^{[\mathrm{c}]}$, \\ Jéssica Magero $^{[\mathrm{d}]}$, Helio Radke Bittencourt ${ }^{[\mathrm{e}]}$
}

[a] Médico veterinário, Mestre em Zootecnia, Faculdade de Medicina Veterinária (ULBRA), Canoas, RS - Brasil, e-mail: carlosgott@cpovo.net

[b] Médico veterinário, Mestrando do PPG em Zootecnia (UFRGS), Porto Alegre, RS - Brasil, e-mail: leonardocanellas@hotmail.com

[c] Graduando do curso de Medicina Veterinária (ULBRA), Bolsista de Iniciação Científica da Faculdade de Medicina Veterinária (PROICT/ULBRA), Canoas, RS - Brasil, e-mail: almeida.m.r@bol.com.br

[d] Graduanda do Curso de Medicina Veterinária (ULBRA), Canoas, RS - Brasil, e-mail: jeh_273@hotmail.com

[e] Estatístico, Mestre em Sensoriamento Remoto e professor assistente do Departamento de Estatística (PUCRS), Porto Alegre, RS - Brasil, e-mail: heliorb@pucrs.br

\section{Resumo}

O trabalho objetivou analisar a taxa de mortalidade (TM) e a causa mortis de 15.466 bovinos de corte de diferentes faixas etárias entre os anos de 2001 e 2007, em uma propriedade rural, com finalidade de recria e de terminação de machos, sendo 4.012 animais na faixa do desmame até 1 ano (G0-1); 4.580 de 1 a 2 anos (G1-2); 4.264 de 2 a 3 anos (G2-3); e 2.610 de 3 a 4 anos de idade (G3-4). Os dados demonstraram que as causas de morte foram: furto (FUR), abscesso (ABS), acidente (ACD), castração (CAS), causas indefinidas (IND), clostridioses (CLS), eletrocussão por raio (RAI), intoxicação (INT), rinotraqueíte infecciosa bovina (IBR), tristeza parasitária bovina (TPB) e verminose/fraqueza (VER). A TM foi maior no G0-1 e G1-2 (1,6\% e 1,3\%) em relação a G2-3 e G3-4 (0,6\% e 0,5\%, respectivamente) (p < 0,01). As causas de morte em cada grupo foram: G0-1 (ACD 12,3\%, IND 27,7\%, IBR 10,8\%, TPB 13,8\%, VER 16,9\%), G1-2 (IND 41,0\%, IBR 8,2\%, TPB 21,3\%, VER 11,5\%), G2-3 (IND 59,3\%, RAI 11,1\%, IBR 11,1\%, TPB 14,8\%), G3-4 (IND 33,3\%, TPB 41,7\%). Concluiu-se que o percentual de mortes por causas indefinidas realça as dificuldades de diagnóstico e de controle a campo da causa mortis nos bovinos de corte e que a taxa de mortalidade reduziu-se com o aumento da idade.

Palavras-chave: Taxa de mortalidade. Causas de morte. Bovinos. Recria. Terminação. 


\begin{abstract}
This article analyzed the mortality rate (MR) and death causes of 15.466 cattle beef of different age between 2001 and 2007. The animals belonged to a propriety that realizes rearing and finishing of males. Four groups were formed: (G0-1) - 4,012 animals of wean until 1 year old; (G1-2) - 4,580 animals of 1 year old until 2 years old; (G2-3) - 4,264 animals of 2 until 3 years old; and (G3-4) - 2,610 animals of 3 until 4 years old. The death causes were: abscess $(A B S)$, accident $(A C D)$, castration (CAS), cattle raiding (CR), clostridiosis (CLS), electrocution by ray (RAY), infectious bovine rhinotracheitis (IBR), intoxication (INT), tick fever (TF), undefined causes (UND) and worms/ weakness (WOR). The MR was higher to G0-1 and G1-2 (1.6\% and 1.3\%) than the G2-3 and G3-4 (0.6\% e 0.5\%, respectively) $(p<0.01)$. The main death causes to each group were: G0-1 (ACD 12.3\%, IBR 10.8\%, TF $13.8 \%$, UND $27.7 \%$, WOR 16.9\%), G1-2 (IBR 8.2\%, TF 21.3\%, UND 41.0\%, WOR 11.5\%), G2-3 (IBR 11.1\%, RAY 11.1\%, TF $14.8 \%$, UND 59.3\%), G3-4 (TF 41.7\%, UND 33.3\%). The percentage of death to undefined causes expresses the difficulty to control the mortality causes in beef cattle on field. The MR decreased with the increase of age.
\end{abstract}

Keywords: Mortality rate. Death causes. Cattle. Rearing. Finishing.

\title{
Introdução
}

Sistemas de produção pecuários devem ser monitorados por meio de indicadores de produtividade, tais como as taxas de natalidade, desmame e mortalidade. A determinação de indicadores permite um monitoramento mais preciso do desempenho biológico e econômico dos sistemas de produção. Os indicadores gerados nas propriedades devem ser comparados entre anos e entre fazendas, permitindo a tomada de decisão adequada e ações corretivas (GOTTSCHALL, 2008). Smith et al. (2001) destacam a taxa de mortalidade como um dos principais índices a serem conhecidos dentro de um sistema de produção eficiente. Corrêa et al. (2001) afirmam que a elevada mortalidade dos rebanhos é um dos fatores que contribuem para a baixa eficiência produtiva característica dos rebanhos gaúcho e brasileiro. Isso ocorre graças à interação existente entre os diferentes fatores que compõem o sistema de produção (BERET'TA et al., 2001), ou seja, uma taxa de mortalidade elevada afeta outros índices produtivos importantes, como, por exemplo, a produção de quilo de peso vivo, refletindo em menor eficiência produtiva e menor retorno econômico. Conforme Vieira et al. (2005) relatam, animais mais jovens tendem a apresentar maior suscetibilidade a enfermidades que levam à morte em relação a animais adultos. São inúmeras as causas de mortes em bovinos, destacando-se entre elas a ocorrência de rinotraqueíte infecciosa bovina, toxi-infecções por bactérias do gênero Clostridium, verminose/ diarreias e tristeza parasitária bovina (VALLE et al., 1998). Essas enfermidades, entre outras, apresentam relevância e grande importância econômica nos rebanhos de bovinos de corte, pois ocasionam queda no desempenho animal (perda de peso) e taxas variáveis de morbidade e mortalidade. Em função da escassez de dados disponíveis na literatura sobre a taxa de mortalidade (TM) em bovinos de corte e suas causas, este trabalho teve por objetivo analisar os índices em uma propriedade rural que realiza recria e terminação de machos.

\section{Material e métodos}

Os registros zootécnicos analisados neste estudo foram obtidos entre os anos de 2001 e 2007, em uma propriedade particular situada no município de Cachoeira do Sul, RS. A propriedade realiza recria e terminação de machos, sendo o rebanho composto por animais recém-desmamados (6-8 meses) até 4 anos de idade. Foram coletados dados de 15.466 animais de diferentes idades, sendo 4.012 animais do desmame até 1 ano (G0-1); 4.580 de 1 a 2 anos (G1-2); 4.264 de 2 a 3 anos (G2-3); e 2.610 de 3 a 4 anos de idade (G3-4). Os dados foram obtidos de animais a partir do desmame até o abate. O manejo nutricional dos animais consistia no uso de campo nativo como o principal recurso forrageiro associado ao uso de pastagens cultivadas no verão 
(milheto - Pennisetum americanum) e no inverno (azevém - Lolium multiflorum), além de alimentação suplementar estratégica com volumoso e/ou concentrado (silagem de milho e resíduos agroindustriais) conforme categoria e exigência dos animais. O manejo sanitário seguiu a recomendação padrão da propriedade, com banhos carrapaticidas periódicos conforme grau de infestação e aplicação de vermífugos com alternância de princípios ativos aproximadamente a cada 30-45 dias para os animais do G0-1; a cada 45-60 dias para os animais do G1-2; a cada 60-90 dias para os animais dos G2-3 e G3-4. Todos os animais tiveram livre acesso à mistura mineral completa com 6,3\% de fósforo. As causas de morte verificadas foram furto (FUR), abscesso (ABS), acidente (ACD), castração (CAS), causas indefinidas (IND), clostridioses (CLS), eletrocussão por raio (RAI), intoxicação (INT), rinotraqueíte infecciosa bovina (IBR), tristeza parasitária bovina (TPB) e verminose/fraqueza (VER). O levantamento realizado foi retrospectivo, apoiado nos registros mensais da propriedade. O diagnóstico da causa das mortes era realizado pelo veterinário responsável, com base na sintomatologia da doença e casuística desta dentro do rebanho. Os dados foram tabulados e uma análise de frequência das causas de morte foi realizada. A partir dessas informações foi calculada a taxa de mortalidade, resultado da divisão do número de mortes pelo número total de animais de cada categoria do rebanho. A análise estatística foi realizada pelo software SPSS ${ }^{\circledR}$ 10.0. por meio do teste Qui-quadrado comparando-se causas de mortalidade e as diferenças entre os grupos (idade).

\section{Resultados}

$\mathrm{Na}$ Tabela 1 são apresentados os dados referentes ao número de mortes $(\mathrm{M})$ e à taxa de mortalidade (TM) conforme o ano (2001 a 2007) e a faixa etária dos animais (G0-1, G1-2, G2-3 e G3-4). Observa-se que, ao fim dos sete anos de coleta de dados, a TM foi maior para os grupos G0-1 e G1-2 ( $<<0,01)$ em relação aos grupos G2-3 e G3-4, que não diferiram entre si ( $p>0,05)$. Os resultados mostraram tendência de maior TM para animais mais jovens, respectivamente 1,6\%, 1,33\% ( $\mathrm{p}<0,01), 0,6 \%$ e $0,5 \%$ ( $>>0,05)$ para G0-1, G1-2, G2-3 e G3-4 (Tabela 1). As maiores taxas de mortalidade registradas ocorreram para o ano de $2002(\mathrm{p}<0,01)$.

Tabela 1 - Número de mortes (M) e taxa de mortalidade (TM) em bovinos de corte, verificadas no período de 2001 a 2007, distribuídas conforme o ano e a faixa etária dos animais $(\mathrm{n}=15466)$

\begin{tabular}{|c|c|c|c|c|c|c|c|c|c|c|c|c|c|c|c|}
\hline \multirow{2}{*}{ Ano } & \multicolumn{3}{|c|}{ G0-1 } & \multicolumn{3}{|c|}{ G1-2 } & \multicolumn{3}{|c|}{ G2-3 } & \multicolumn{3}{|c|}{ G3-4 } & \multicolumn{3}{|c|}{ Total } \\
\hline & n & $\mathbf{M}$ & TM & $\mathrm{n}$ & $\mathbf{M}$ & TM & $\mathrm{n}$ & $\mathbf{M}$ & TM & $\mathrm{n}$ & $\mathbf{M}$ & TM & $\mathrm{n}$ & $\mathbf{M}$ & TM \\
\hline 2001 & 814 & 11 & $1,4 \% \mathrm{~A}$ & 604 & 16 & $2,7 \% \mathrm{~B}$ & 513 & 2 & $0,4 \% \mathrm{~A}$ & 525 & 1 & $0,2 \% \mathrm{~A}$ & 2456 & 30 & $1,2 \% \mathrm{~A}$ \\
\hline 2002 & 660 & 29 & $4,4 \% B$ & 813 & 23 & $2,8 \% \mathrm{~B}$ & 584 & 5 & $0,9 \% \mathrm{~A}$ & 359 & 5 & $1,4 \% \mathrm{~A}$ & 2416 & 62 & $2,6 \% B$ \\
\hline 2003 & 600 & 9 & $1,5 \% \mathrm{~A}$ & 594 & 4 & $0,7 \% \mathrm{~A}$ & 596 & 2 & $0,3 \% \mathrm{~A}$ & 343 & 1 & $0,3 \% \mathrm{~A}$ & 2133 & 16 & $0,8 \% \mathrm{~A}$ \\
\hline 2004 & 600 & 8 & $1,3 \% \mathrm{~A}$ & 590 & 3 & $0,5 \% \mathrm{~A}$ & 589 & 9 & $1,5 \% \mathrm{~A}$ & 338 & 3 & $0,9 \% \mathrm{~A}$ & 2117 & 23 & $1,1 \% \mathrm{~A}$ \\
\hline 2005 & 600 & 4 & $0,7 \% \mathrm{~A}$ & 593 & 2 & $0,3 \% \mathrm{~A}$ & 589 & 2 & $0,3 \% \mathrm{~A}$ & 266 & 1 & $0,4 \% \mathrm{~A}$ & 2048 & 9 & $0,4 \% \mathrm{~A}$ \\
\hline 2006 & 303 & 2 & $0,7 \% \mathrm{~A}$ & 806 & 7 & $0,9 \% \mathrm{~A}$ & 587 & 2 & $0,3 \% \mathrm{~A}$ & 433 & 0 & $0,0 \% \mathrm{~A}$ & 2129 & 11 & $0,5 \% \mathrm{~A}$ \\
\hline 2007 & 435 & 2 & $0,5 \% \mathrm{~A}$ & 580 & 6 & $1,0 \% \mathrm{~A}$ & 806 & 5 & $0,6 \% \mathrm{~A}$ & 346 & 1 & $0,3 \% \mathrm{~A}$ & 2167 & 14 & $0,7 \% \mathrm{~A}$ \\
\hline Total & 4012 & 65 & $1,6 \% 0^{\mathrm{a}}$ & 4580 & 61 & $1,33 \% \%^{a}$ & 4264 & 27 & $0,6 \% 0^{\mathrm{b}}$ & 2610 & 12 & $0,5 \%{ }^{\mathrm{b}}$ & 15466 & 165 & $1,1 \%$ \\
\hline
\end{tabular}

Legenda: a,b: Médias na mesma linha seguidas de letras diferentes representam diferença estatística significativa $(\mathrm{p}<0,01)$;

A,B: Médias na mesma coluna seguidas de letras diferentes representam diferença estatística significativa $(p<0,01)$;

G0-1: Grupo de animais do desmame até 1 ano de idade; G1-2: Grupo de animais de 1 até 2 anos de idade; G2-3: Grupo de animais de 2 até 3 anos de idade; G3-4: Grupo de animais de 3 até 4 anos de idade.

Rev. Acad., Ciênc. Agrár. Ambient., Curitiba, v. 8, n. 3, p. 327-332, jul./set. 2010 
Na Tabela 2 estão disponíveis os percentuais das causas de mortes diagnosticadas nas diferentes faixas etárias. Observando-se a Tabela 2, nota-se que as causas indefinidas (IND) apresentaram o maior percentual total entre todas as causas possíveis de morte, $38,2 \%$, ao passo que mortes por rinotraqueíte infecciosa bovina (IBR), tristeza parasitária bovina (TPB) e verminose (VER) apresentaram 9,1\%, 18,8\% e 11,5\%, respectivamente. Para cada faixa etária, a taxa de mortalidade para causas indefinidas foi: 27,7\% para G0-1, 41\% para G1-2, 59,3\% para G2-3 e 33,3\% para G3-4. No grupo etário G0-1 (Tabela 2), constatou-se que as causas prevalentes de morte definida referem-se à: rinotraqueíte infecciosa bovina (IBR), tristeza parasitária bovina (TPB) e verminose (VER), respectivamente, 10,8\%, 13,8\% e 16,9\%. Neste mesmo grupo etário foi constatada maior vulnerabilidade a acidentes, $(\mathrm{ACD}=12,3 \%)$.

Tabela 2 - Percentual de mortes (\%) verificadas no período de 2001 a 2007, conforme a causa e grupo etário de bovinos de corte

\begin{tabular}{|c|c|c|c|c|c|c|c|c|c|c|}
\hline \multirow{2}{*}{ Causa } & \multicolumn{2}{|c|}{ G0-1 } & \multicolumn{2}{|c|}{ G1-2 } & \multicolumn{2}{|c|}{ G2-3 } & \multicolumn{2}{|c|}{ G3-4 } & \multicolumn{2}{|c|}{ Total } \\
\hline & $\mathrm{n}$ & $\%$ & $\mathbf{N}$ & $\%$ & $\mathbf{N}$ & $\%$ & $\mathbf{N}$ & $\%$ & $\mathrm{n}$ & $\%$ \\
\hline FUR & 2 & 3,1 & 1 & 1,6 & 0 & 0 & 0 & 0 & 3 & 1,8 \\
\hline ABS & 0 & 3,3 & 2 & 0 & 0 & 0 & 0 & 0 & 2 & 1,2 \\
\hline ACD & 8 & 12,3 & 2 & 3,3 & 0 & 0 & 1 & 8,3 & 11 & 6,7 \\
\hline CAS & 0 & 0 & 0 & 0 & 1 & 3,7 & 0 & 0 & 1 & 0,6 \\
\hline CLS & 4 & 6,2 & 2 & 3,3 & 0 & 0 & 0 & 0 & 6 & 3,6 \\
\hline RAI & 1 & 1,5 & 1 & 1,6 & 3 & 11,1 & 0 & 0 & 5 & 3 \\
\hline IND & 18 & 27,7 & 25 & 41 & 16 & 59,3 & 4 & 33,3 & 63 & 38,2 \\
\hline INT & 5 & 7,7 & 3 & 4,9 & 0 & 0 & 1 & 8,3 & 9 & 5,5 \\
\hline IBR & 7 & 10,8 & 5 & 8,2 & 3 & 11,1 & 0 & 0 & 15 & 9,1 \\
\hline ТРB & 9 & 13,8 & 13 & 21,3 & 4 & 14,8 & 5 & 41,7 & 31 & 18,8 \\
\hline VER & 11 & 16,9 & 7 & 11,5 & 0 & 0 & 1 & 8,3 & 19 & 11,5 \\
\hline Total & 65 & 100 & 61 & 100 & 27 & 100 & 12 & 100 & 165 & 100 \\
\hline
\end{tabular}

Legenda: FUR: furto; ABS: abscesso; ACD: acidente; CAS: castração; CLS: clostridioses; RAI: eletrocussão por raio; IND: causas indefinidas; INT: intoxicação; IBR: rinotraqueíte infecciosa bovina; TPB: tristeza parasitária bovina e VER: verminose/fraqueza; G0-1. Grupo de animais do desmame até um ano de idade; G1-2. Grupo de animais de um até dois anos de idade; G2-3. Grupo de animais de dois até três anos de idade; G3-4. Grupo de animais de três até quatro anos de idade.

Nos outros grupos as principais causas definidas de morte foram, para os seus respectivos grupos, as seguintes: $8,2 \%$ IBR, 21,3\% TPB e 11,5\% VER para G1-2; 11,1\% RAI, 11,1\% IBR e 14,8\% TPB para G2-3; 41,7 \% TPB para G3-4.

\section{Discussão}

Poucos trabalhos da literatura científica apresentam dados sobre os índices de mortalidade em bovino de corte. Gottschall (2008) relata taxas de mortalidade inferiores a 1\% em animais em terminação, apresentando 
indicadores de mortalidade em sistemas de recria e terminação variáveis entre $0,36 \%$ e $2,46 \%$. Pötter et al. (2000) relataram TM em rebanhos de ciclo completo, conduzidos de forma extensiva, de 8\%, 4\%, 3\% e 2\%, respectivamente para bezerros, animais de 1 a 2 anos, de 2 a 3 anos e de 3 a 4 anos de idade. Esses resultados demonstram as mesmas tendências apresentadas neste trabalho, em que G0-1 e G1-2 tiveram maiores TM em relação a animais mais idosos (Tabela 1). Ollhoff et al. (2008), em rebanhos leiteiros, destacaram que os animais recém-nascidos são mais suscetíveis às doenças infecto-contagiosas. Arruda e Corrêa (1992) consideram 5\% uma TM aceitável para bezerros de corte. Eles obtiveram, em um período avaliativo de quatro anos num rebanho de ciclo completo, médias da taxa de mortalidade para animais de 0 a 1 ano, 1 a 2 anos, 2 a 3 anos de idade, vacas e touros, de respectivamente 4,9\%, 2,4\%, 0,4\%, 1,0\%, 3,1\%. Corrêa e Arruda (1988), avaliando índices zootécnicos de um rebanho de ciclo completo por um período de cinco anos, recomendam como taxas de mortalidade aceitáveis $8 \%, 4 \%, 2 \%, 3 \%$ e 1\% respectivamente para animais de 0 a 1 ano, de 1 a 2 anos, 2 a 3 anos de idade, vacas e touros. Cezar e Euclides Filho (2000) relataram taxas de mortalidade aceitáveis para bezerros e animais acima de 1 ano de idade, de $4 \%$ e $1 \%$, respectivamente. Relatos da literatura vêm corroborar os obtidos no presente estudo, indicando que animais de até 2 anos de idade apresentam maiores taxas de mortalidade. Isso reflete a maior vulnerabilidade dos animais jovens às doenças infecto-contagiosas, parasitárias e tóxicas. Vieira et al. (2005) observaram taxas de mortalidade de $6 \%$ e de $1 \%$, respectivamente, para bezerros e animais adultos criados em sistema extensivo. Ainda nesta pesquisa fatores isolados não identificados, tais como procedimentos durante as vacinações, efeitos climáticos, mudança de funcionários podem ter interferido sobre os resultados. O elevado percentual de causas indefinidas (IND) de mortalidade, apresentado na Tabela 2, pode ser associado às dificuldades de realização de um diagnóstico preciso, graças a eventuais falhas ou atrasos na identificação de alguns dos animais mortos. O grupo etário G0-1 apresentou maior percentual de acidentes em relação às demais idades, vulnerabilidade esta que provavelmente está relacionada à maior fragilidade física e imaturidade dos animais desta faixa etária. Ollhoff et al. (2008), investigando um rebanho leiteiro, ressaltaram a necessidade de inspecionar o ambiente para averiguar as possíveis causas dos acidentes. Esta prática de inspeção regular também é importante à bovinocultura de corte. Os resultados dos grupos etários G1-2, G2-3 e G3-4 (Tabela 2) demonstraram que as causas definidas de morte, rinotraqueíte infecciosa bovina (IBR) e verminose (VER), diminuíram suas incidências com o aumento da idade. Todavia, as mortes causadas por tristeza parasitária bovina (TPB) mantiveram altos percentuais em relação às outras causas definidas dos respectivos grupos etários, explicados pelos distintos fatores que estão ligados à etiologia da TPB (GONÇALVES, 2000). Segundo Radostits et al. (2000), todas as idades e raças são suscetíveis ao vírus da rinotraqueíte bovina (IBR), sendo mais corrente em animais acima de 6 meses de idade, em virtude da diminuição da imunidade maternal adquirida. Os autores ainda afirmaram que animais jovens foram os mais suscetíveis às verminoses, e com menor freqüência, animais adultos são infestados anualmente.

\section{Conclusão}

Em função dos dados levantados, pode-se concluir que há elevado percentual de mortes nos bovinos de corte por causas indefinidas, ressaltando-se as significativas dificuldades vivenciadas nas fazendas pecuárias, impedindo um controle mais satisfatório desta variável; e que a taxa de mortalidade foi reduzindo-se com o aumento da idade dos animais.

\section{Referências}

ARRUDA, Z. J. de; CORRÊA, E. S. Avaliação técnico-econômica de sistemas de produção de gado de corte: o sistema físico de produção do CNPGC. Campo Grande: EMBRAPA-CNPGC, 1992. EMBRAPA-CNPGC. Comunicado Técnico, 42. 
BERETTA, V.; LOBATO, J. F. P.; MIELITZ NETTTO, C. G. A. Produtividade e eficiência biológica de sistemas pecuários de cria diferindo na idade das novilhas ao primeiro parto e na taxa de natalidade do rebanho no Rio Grande de Sul. Revista Brasileira de Zootecnia, v. 30, n. 4, p. 1278-1286, 2001.

CEZAR, I. M.; EUCLIDES FILHO, K. Sistema de produção de novilho precoce: avaliação bioeconômica. In: ENCONTRO NACIONAL DO NOVILHO PRECOCE, 5., 2000, Campo Grande, MS. Anais... Campo Grande, MS: Encontro Nacional Do Novilho Precoce, 2000. Disponível em: < http://www.cnpgc.embrapa.br/eventos/2000/ novilhoprecoce/precoce.html>. Acesso em: 15 ago. 2009.

CORRÊA, E. S.; ARRUDA, Z. J. Avaliação preliminar do sistema de produção de gado de corte implantado no CNPGC, período: 1983/84 a 1986/87. Campo Grande: EMBRAPA-CNPGC, 1988. EMBRAPA-CNPGC. Documentos, 38 .

CORRÊA, E. S. et al. Desempenho reprodutivo em um sistema de produção de gado de corte. Campo Grande: EMBRAPA-CNPGC, 2001. EMBRAPA-CNPGC. Documentos, 13.

GONÇALVES, P. M. Epidemiologia e controle da tristeza parasitária bovina na região sudeste do Brasil. Revista Ciência Rural, v. 30, n. 1, p. 187-194, 2000.

GOT'TSCHALL, C. S. Indicadores de produtividade em rebanhos bovinos de corte e de leite. In: Sociedade Brasileira de Medicina Veterinária. (Org.). Bovinocultura - PROMEVET - Programa de atualização em medicina veterinária. Porto Alegre: Artmed Panamericana, 2008. v. 1. p. 11-49.

OLLHOFF, R. D. et al. Causas de descarte e óbito de bovinos leiteiros entre 2000-2006 em um rebanho de alta produção. Revista Acadêmica: Ciências agrárias e ambientais, v. 6, n. 3, p. 381-387, 2008.

PÖTTER, L.; LOBATO, J. F. P.; MIELITZ NETTTO, C. G. A. Análise econômica de modelos de produção com novilhas de corte primíparas aos dois, três e quatro anos de idade. Revista Brasileira de Zootecnia, v. 29, n. 3, p. 861-870, 2000.

RADOSTITS, O. M. et al. Veterinary medicine. 9th ed. New York: W. B. Saunders, 2000.

SMITH, R. A. et al. Health and production management in beef feeddlots. In: RADOSTITS, O. M. Herd health: food animal production medicine. 3rd ed. Philadelphia: W. B. Saunders Company, 2001. p. 581-634.

VALLE, E. R.; ANDREOT'TI, R.; THIAGO, L. R. L. Estratégias para aumento da eficiência reprodutiva e produtiva em bovinos de corte. Campo Grande: EMBRAPA-CNPGC, 1998. EMBRAPA-CNPGC. Documentos, 71.

VIEIRA, A. et al. Desempenho produtivo nas fases de cria e recria em um sistema de produção de gado de corte no Brasil Central. Campo Grande: EMBRAPA-CNPGC, 2005. EMBRAPA-CNPGC. Documentos, 18.

Recebido: 16/09/2009

Received: 09/16/2009

Aprovado: $12 / 05 / 2010$ Approved: 05/12/2010 